This item was submitted to Loughborough's Institutional Repository (https://dspace.lboro.ac.uk/) by the author and is made available under the following Creative Commons Licence conditions.

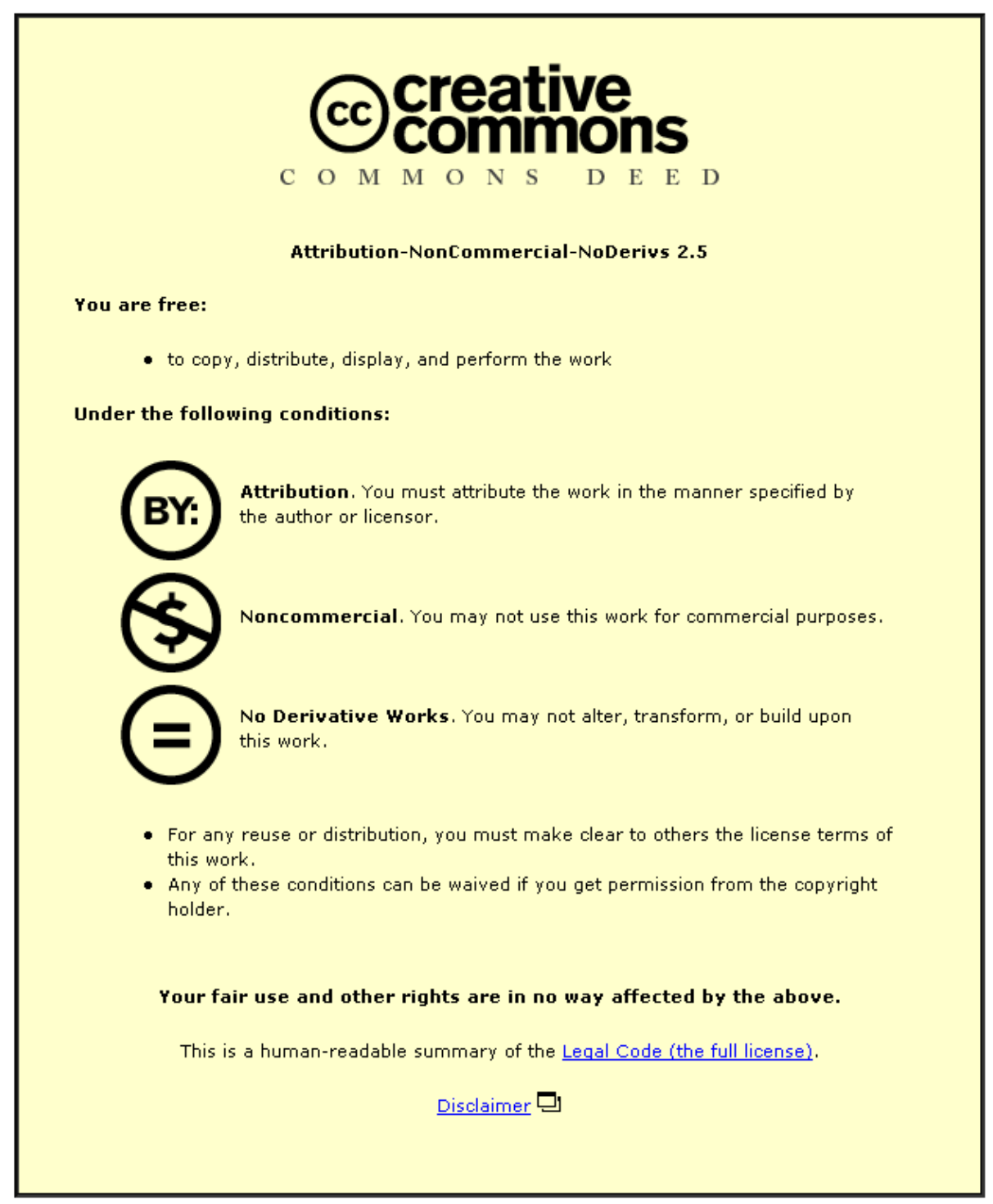

For the full text of this licence, please go to: http://creativecommons.org/licenses/by-nc-nd/2.5/ 


\title{
Transmission illuminance proxy HDR imaging: A new technique to quantify luminous flux
}

\author{
*†J. Mardaljevic PhD, †B. Painter PhD and $\ddagger$ M. Andersen PhD \\ $\dagger$ Institute of Energy and Sustainable Development \\ De Montfort University \\ The Gateway, Leicester, LE1 9BH, UK \\ $\ddagger$ Massachusetts Institute of Technology \\ MIT Room 5-418 \\ 77 Massachusetts Avenue, Cambridge \\ MA 02139-4307, USA \\ *Corresponding author jm@dmu.ac.uk \\ Phone: +44 (0) 1162577972 \\ Fax: +44 (0) 1162577981
}

\begin{abstract}
A technique to measure arbitrarily complex luminous fluxes across large areas is presented. The technique is founded on high dynamic range imaging technology and can be achieved using a standard consumer digital camera and everyday materials such as printer-grade white paper. The same approach can also be used to determine the direct and diffuse components of illuminance. The technique has been named transmission illuminance proxy - high dynamic range imaging or TIP-HDRI.
\end{abstract}

Keywords: Illuminance measurement, high dynamic range images, luminous flux, light-pipes

\section{Introduction}

The authors describe a new technique to measure luminous flux and illuminance using high dynamic range imaging. The pixel data in a high dynamic range (HDR) image contain real-world luminance values equivalent to those that might be measured using a spot photometer. There are a small number of specialist HDR cameras on the market, however it is possible to create 
HDR images from multiple exposures taken by consumer digital cameras [1]. For the technique described in this paper, the luminance values in the HDR image serve as a proxy measurement for incident illuminance. The approach is called transmission illuminance proxy - high dynamic range imaging or TIP-HDRI. It is suited to determining the luminous flux through building apertures such as windows, facade systems, light-pipes etc. The HDRI-based approach allows rapid quantification of the luminous flux in light-fields of arbitrary complexity where the standard measurement practices would be either time-consuming or impossible to apply with any certainty due to the practical difficulties of carrying out numerous spot measurements covering large areas under natural (i.e. non-steady) conditions. The following applications of transmission illuminance proxy - high dynamic range imaging are described in this paper:

- Determination of the distribution and quantity of luminous flux through a window aperture.

- Quantification of the luminous flux emerging from a light-pipe.

- A simple device to measure direct (i.e. solar) and diffuse (e.g. sky) horizontal illuminances.

The examples are followed by outlines for a number of possible speculative applications. The new technique is presented as a proof-of-concept study. Results from limited testing of the accuracy of predictions in one of the application areas is given. There is a discussion of a more rigourous and complete validation that the authors intend to carry out in the near future.

\section{Background}

Although TIP-HDRI is a generalised approach for measuring luminous flux through any aperture, the background to the technique is described in terms of the long-standing problem of determining the quantity of light entering through a window. This is usually determined from one or more measurements of illuminance taken, for example, on the inside surface of a window [2]. If the window is set flush with the external facade, and there are no nearby structures such as projections or shading devices, then the luminous flux across the glazing will have a uniform distribution. If, however, there is any shading of the glazing then the flux distribution through it will become non-uniform. The greater the propensity for partial shading of the window, the greater will be the degree of non-uniformity in the flux. External shading devices are now a common feature of buildings in many parts of the world. These are designed to either minimise direct solar radiation, improve the provision of diffuse daylight, or some mixture of the two. With these shading devices, even under overcast conditions, it may require a fairly large number of measurements across the glazing to obtain a reliable average illuminance as a basis for determining the luminous flux under real-world conditions. Under 
clear sky conditions the shading devices can produce large illuminance gradients over small spatial scales and across large areas of the glazing. This could render the task of determining an average illuminance impractical or wellnigh impossible as the illumination conditions will be continually changing during the time it will take to carry out the measurements.

The TIP-HDRI technique can determine the luminous flux through windows regardless of the complexity of the illuminance field incident on the window. The technique is based on determining the relation between the luminance of a diffusing surface viewed from the "rear" and the illuminance incident on the "front" side. When this is known, the incident flux on the front-side of the diffusing surface can be derived from measurements of the luminance taken from the rear-side. Of course, if the luminances are recorded by a spot-photometer, then multiple measurements have to be made and there is little advantage over using an illuminance meter. The essential feature of the technique proposed here is that the luminance across the entire surface of the diffusing material is captured using a camera-based approach. The resulting image is a data map containing an accurate measurement of luminance at every pixel of the image [1]. Thus it is possible to derive an incident illuminance on the front-side of the diffusing material for every pixel in the image. In other words, one million "point" illuminance values can be derived from a one mega-pixel luminance image of the diffusing material. It is then a straightforward matter to derive integrated flux values for arbitrarily complex illuminance fields using simple image processing techniques. This is the basic principle of the transmission illuminance proxy - high dynamic range imaging technique.

\subsection{Theory}

Consider the flow of light as a luminous flux having arbitrary directional components, e.g. diffuse and direct daylight entering a space through a window aperture, Figure 1a. A diffusing material placed across the aperture receives luminous flux $E$. The diffusing material will reflect, absorb and transmit the light to varying degrees depending on the nature of the incident luminous flux and the properties of the diffusing material, Figure 1b. The incident flux will produce in the diffusing material a luminance $L$ that will vary in some proportion to the magnitude and direction(s) of the incident luminous flux. If the relation between the incident flux and the resulting luminance in the diffusing material can be determined, then it becomes a relatively simple matter to derive incident luminous flux from measurements of the luminance of the diffusing material.

A first approximation is to assume a proportional relation between the front-side incident illuminance $E$ and the rear-side visible luminance $L$ such that:

$$
L=q E
$$

Where $q$ is the luminance coefficient for the transmission of visible radiation through the diffusing material. The luminance coefficient has units of 
$\mathrm{cd} \mathrm{m}^{-2} \mathrm{lux}^{-1}$ or $\mathrm{sr}^{-1}$ [3]. It is then a straightforward task to calibrate the diffusing material to determine $q$ by taking measurements of the incident illuminance $E$ and the rear-side visible luminance $L$. Once this has been done, the flux incident on the diffusing material can de determined from measurements of luminance on the rear-side.

This approximation can give reasonable accuracy depending on the character of the incident illumination and the viewing conditions on the rear-side of the diffusing material. However, a little experimentation reveals that the luminance coefficient $q$ depends on the direction of the incident illumination when it is non-uniform, e.g. when there is direct sun. Furthermore, the viewing conditions on the rear-side may be such that a measurable fraction of the luminance of the diffusing material results from luminous flux that is reflected off the rear-side of the diffusing material. In the more general case, the luminance $L_{s}$ "seen" on the rear-side of the diffusing material can be composed of up to three components:

1. The luminance $L_{i}$ resulting from the scattering and transmission of incident, diffuse (i.e. isotropic) illumination.

2. The luminance $L_{\theta}$ resulting from the transmission/scattering of incident, directional illumination - the beam at angle $\theta$ to the surface normal.

3. The luminance $L_{r}$ resulting from the reflection of illumination on the rear-side surface of the diffusing material.

This is illustrated in Figure 2. The "seen" luminance $L_{s}$ is simply the sum of the three components:

$$
L_{s}=L_{i}+L_{\theta}+L_{r}
$$

The two components of luminance due to transmission are related to the incident illuminance as given in Equation 1:

$$
\begin{aligned}
E_{i} & =L_{i} / q_{i} \\
E_{\theta} & =L_{\theta} / q_{\theta}
\end{aligned}
$$

only now the luminance coefficient $q_{\theta}$ for the directional beam has a dependance on the angle of incidence $\theta$. The magnitude of the rear-side incident illumination $E_{r}$ is not needed, however the luminance $L_{r}$ resulting from it will need to be determined from measurement. Experimentally, it is far more convenient to measure and correct for $L_{r}$ than it is to attempt to eliminate it by means of light-proofing the measurement zone on the rear-side of the diffusing material (additionally, all surfaces on the inside would need to be black). If the luminance coefficients $q_{i}$ and $q_{\theta}$ are known, then the directional and diffuse incident illuminances $E_{\theta}$ and $E_{i}$ can be determined from measurements of $L_{\theta}$ and $L_{i}$. 


\section{$2.2 \quad$ A suitable diffusing material}

The diffusing material should, ideally, be colour-neutral and behave as a perfect diffuser for both the transmission and the reflectance of visible radiation. Various plastic materials such as the opalescent sheets used as diffusers in light-boxes were considered. These materials however typically had marked specular reflective properties or were not readily available in large sizes. Following a little experimentation it was discovered that good results can be obtained using materials as commonplace as high-quality white paper. For all the examples described below, standard wide-format ink-jet paper was used. This material also has the advantage of being cheap and readily available in large sizes.

On close inspection it is apparent that, at scales of the order of a $\mathrm{mm}$ or so, paper is an inhomogeneous diffuser of light. In reality therefore, the luminance coefficients for any fixed lighting conditions will be varying over these small scales. When taking images of the paper with a digital camera it is likely that the effective pixel dimension will be sufficiently small to resolve the small inhomogeneities. The paper inhomogeneity could influence the results only if illuminance values at a "point" were derived from areas of paper markedly smaller than the size of a photocell head, i.e. less than about $20 \mathrm{~mm}^{2}$. In practice, "point" illuminance values should be derived from a collection of pixels over a sufficient area rather than a single pixel (see example in Section 4.3). For the majority of applications it is the flux across large areas that is of interest rather than point values and so the paper inhomogeneity will not have any direct bearing.

\section{Measurements of the luminance coefficient of paper}

Measurements to determine the luminance coefficient of the diffusing material can be carried out employing varying degrees of experimental rigour. The minimum light measuring equipment needed comprises just an illuminance meter and a luminance meter. For a diffuse field of incident illumination, measurements are taken of the incident illuminance $E_{i}$ and the (uncorrected) luminance of the paper $L_{i}^{\prime}$. For each change of illumination conditions, a measurement of the luminance due to reflection $L_{r}$ is made. The luminance $L_{i}$ resulting from just the transmission of diffuse illumination (i.e. the corrected value) is simply:

$$
L_{i}=L_{i}^{\prime}-L_{r}
$$

In practice, precise isotropic diffuse illumination conditions are difficult to achieve. They can however be approximated by a variety of means, e.g. shining a diffused projector beam into a box that has a uniform diffuse white finish. Measurements can also be made under steady, ambient daylit conditions. 
For directional beams, measurements taken at incident angles $0^{\circ}$ to $80^{\circ}$ in increments of $10^{\circ}$ should be sufficient. Commonplace data projectors can be used since they provide a steady directional beam.

At the time that the TIP-HDRI approach was first formulated and tested, the first two authors (Mardaljevic and Painter) had only limited experience of light measurement and that using fairly standard equipment, e.g. illuminance meters and spot photometers. In contrast, Andersen, who has pioneered novel techniques to characterise the complex transmission properties of non-standard glazings, has considerable experience of carrying out exacting measurements under controlled conditions. Since it was hoped that the TIPHDRI approach, including basic calibration, would be available to potential users with only modest equipment requirements, it was decided to compare calibration measurements taken under different degrees of rigour. Thus the measurements needed to derive the luminance coefficient of the paper were taken in ambient conditions by relative novices (Mardaljevic and Painter) and later, for comparison, in controlled conditions by an expert (Andersen).

\section{1 'Novice' set: measurement under ambient light conditions}

Measurements under diffuse illumination were taken in a large space during steady clear sky conditions, and for a range of illuminance values measured at the plane of the paper. The apparatus was arranged so that there were no directly illuminated surfaces anywhere near the paper. This we assumed to provide approximately diffuse illumination conditions for the measurement of the luminance coefficient. The following measurements were taken:

- the illuminance incident on the front-side plane of the paper;

- the luminance of the paper; and,

- the luminance of the paper 'behind' a black opaque patch.

The illuminance was monitored during each sequence of measurements to ensure stable conditions.

For the direct measurements, illumination was provided by the light beam from a standard data projector, i.e. those commonly used for presentations. As with the diffuse illumination, measurements were made in a daylit workspace under ambient daylight conditions on a clear sky day to ensure stability. The diffuse components of luminance and illuminance were measured separately and subtracted from the totals to give the values due to direct illumination only. Checks were made to ensure that the measurement area illuminated by the projector was uniform. The projector was moved over an arc of radius approximately $5 \mathrm{~m}$ to illuminate the paper at angles between $0^{\circ}$ and $80^{\circ}$ in increments of $10^{\circ}$. Orientation and alignment were determined using rudimentary string and protractor techniques. 


\section{2 'Expert' set: measurement in a lighting laboratory under controlled conditions}

A set of photometric measurements was carried out at the Daylighting Laboratory of the Massachusetts Institute of Technology (MIT) to assess the transmission properties of the ink-jet paper in controlled laboratory conditions. A sample of paper from the roll used by Mardaljevic and Painter was sent to Andersen. The transmission properties of the ink-jet paper for diffuse illumination were also assessed using an integrating sphere to generate a hemispherical, uniform illumination of the paper. The experimental set-up is described in detail in the Appendix together with an evaluation of the diffusing properties of the paper.

\subsection{Comparison of the measured values of $q$ for the two data sets}

The luminance coefficients for the 'novice' and 'expert' measurement sets are presented in Figure 3. The incident angles for directional illumination range from $0^{\circ}$ to $80^{\circ}$ in $10^{\circ}$ steps (the points are displaced slightly either side of decade incidence angle marks to avoid overlap). The luminance coefficient for diffuse illumination is shown also (to the left of the $0^{\circ}$ mark on the abscissa). Firstly, the luminance coefficients of the 'novice' set are noticeably lower than corresponding values of the 'expert' set. Relatively few measurements at each angle were taken for the 'novice' set, and so the significance of the error bars for this set should be judged accordingly. The relative difference between the two sets is greatest for diffuse light and normal incidence direct light (approximately 14\%), and gradually diminishes to values between $8 \%$ and $1 \%$ for direct light with angles of incidence $30^{\circ}$ or greater. For direct illumination, the two curves have a very similar form: a gradual reduction in $q$ as the incidence angle increases from $0^{\circ}$ to $30^{\circ}$. Greater than $30^{\circ}$ the fall-off is still less than the cosine law. This observation is consistent with previous measurement by Andersen on the transmission of light through a white opalescent diffusing material: that also diminished more slowly than the cosine law [4].

Note that, for either data set, the luminance coefficient for diffuse light is greater than any of the values for direct light. We would have expected that the value for diffuse light would be some weighted mean of the direct values, since diffuse light is equivalent to direct light arriving from all directions. Significant backscatter by the paper of light back into the integrating sphere could affect the measurement of $q$ under diffuse light for the 'expert' set resulting in a larger than true value. For the 'novice' set, where the illumination of the paper and the measurements were taken in open space, the possible cause may be related to the spectral properties of the various elements involved: spectral sensitivity of the detectors, spectral power output of the light sources (direct and diffuse sources are different) and spectral selectivity of paper in transmission. Given that in absolute terms the differences are small (14\%) and comparable to typical uncertainties found in 
photometry measurements, we suggest that the 'novice' method does indeed provide potentially reliable values for $\mathrm{q}$ and should be considered by those wishing to experiment with TIP-HDRI.

\section{Application examples}

The TIP-HDRI method was used to determine luminous flux and illuminance quantities for a variety of application scenarios. These were:

- The luminous output of a window under diffuse and directly illuminated daylight conditions.

- The luminous output of a light-pipe under directly illuminated daylight conditions.

- The measurement of horizontal direct and diffuse illuminance.

The section concludes with a discussion of speculative applications of the TIP-HDRI technique.

\subsection{Luminous output of a window}

The TIP-HDRI technique was used to determine the luminous flux through a double glazed window set in a moderate reveal with an external overhang. The window is comprised of standard $4 \mathrm{~mm}$ double-glazed clear glass and has dimensions $0.38 \mathrm{~m}$ by $0.98 \mathrm{~m}$. A cut-to-size sheet of paper, with six small pieces of opaque black card on the side adjacent to the glass, was placed over the inside surface of the window, Figure 4a. The patches of opaque card are to determine the rear-side component of reflected luminance. Regardless of the homogeneity or otherwise of the incident illumination, the rear-side reflected component of illuminance tends to exhibit only gradual variation across the paper. Hence only a small number of opaque patches are needed to obtain a reasonable estimate of the rear-side reflected component of illuminance.

A digital SLR camera was used to record a sequence of images for HDR synthesis. The ideal position for the camera is normal to the centre of the window and on a tripod. It should be sufficiently far back so that the distance from the camera to the window is at least five times the maximum linear dimension of the window. It is then reasonable to treat the effective pixel dimension as constant across the image. The sequence of images needed to create a HDR image should be taken in quick succession to minimise the potential for the illuminance conditions to change during the acquisition. An automated process where the camera is controlled by a computer is preferred. All the HDR images used for the experiments described in this paper were captured using the HDRcapOSX program [5]. Manual capture will take significantly longer and it is advised to monitor conditions with an illuminance meter to ensure that stable illumination prevailed during the taking of the images. 
The HDR images created by the HDRcapOSX capture program were loaded into the freely-available Photosphere HDR browser for display and analysis [5]. The Photosphere software acts very much like a browser and cataloguing tool for standard digital images, with the addition that it handles high dynamic range images created either from digital cameras or by rendering (e.g. images produced by the Radiance lighting simulation system). Photosphere can also display the HDR image in false colour with an associated legend to indicate the mapping between luminance and colour, Figures $4 \mathrm{~b}$ and $4 \mathrm{c}$. Figure $4 \mathrm{~b}$ shows a window illuminated by diffuse daylight, and Figure 4c shows an identical window now illuminated with direct sunlight also. The range used for the scales was 3.8 to $140 \mathrm{~cd} \mathrm{~m}^{-2}$ and 100 to $3500 \mathrm{~cd} \mathrm{~m}^{-2}$ for Figures $4 \mathrm{~b}$ and $4 \mathrm{c}$ respectively. These images are best viewed in full colour on-line at the publisher's website.

In addition to the selection of individual pixels, Photosphere has a facility to return the average luminance value for pixels selected within a rectangular area. Thus, provided the image of the window sits square in the "frame", it should be possible to select the entire window area for analysis. The area of the glazing is highlighted in Figures $4 \mathrm{~b}$ and $\mathrm{c}$ with a dashed black-white rectangle. The luminous flux $F$ through the window is given by:

$$
F=E \cdot A=q^{-1}\left(L_{w}-L_{r}\right) \cdot A
$$

where: $E$ is the mean illuminance on the rear-side of the window, $A$ is the area of the glass, $L_{w}$ is the mean luminance of the paper across the window (as "seen" by the camera), $L_{r}$ is the rear-side reflected component of luminance and $q$ is the luminance coefficient for the paper.

The luminous flux is determined as follows. The example using the measurements for the window under diffuse illumination conditions (Figure 4b) is given first. The rear-side reflected component of luminance $L_{r}$ was determined by taking the average value of the luminances of the six opaque patches. This was measured to be $30.3 \mathrm{~cd} \mathrm{~m}^{-2}$. Next, the average luminance $L_{w}$ across the glazing area was determined using the rectangular selection tool, giving $121.7 \mathrm{~cd} \mathrm{~m}^{-2}$. The mean illuminance is simply $L_{w}-L_{r}$ multiplied by the reciprocal of the luminance quotient. Here, the diffuse value from the 'novice' set of measurements (i.e. $q_{i}=0.0448 \mathrm{sr}^{-1}$ ) was used, giving a mean illuminance on the rear-side of the window of 2038 lux. Thus the flux through the window was 759 lumens.

Repeating the procedure for the window under direct sun illumination (Figure 4c), the values were $L_{r}=352 \mathrm{~cd} \mathrm{~m}^{-2}$ and $L_{w}=1871 \mathrm{~cd} \mathrm{~m}^{-2}$. The luminance coefficient used is now that for direct illumination where the angle of incidence of the sunlight to the surface normal of the window was approximately $40^{\circ}$, i.e. $q_{\theta=40}=0.0395 \mathrm{sr}^{-1}$. This gave a lumen output for the window of approximately 14400 lumens. The diffuse illuminance field through the window exhibits moderate inhomogeneity due to the window reveal and nearby self-shading of the building: illuminance at the centre is approximately twice that close to the left-most edge of the glass, Figure $4 \mathrm{~b}$. For the window shown in Figure 4c, the illuminance of the direct 
sun-illuminated lower half of the window was six or more times greater than the diffuse-only upper half.

\subsection{Luminous output of a light-pipe}

A technique similar to that used for the window was employed to determine the luminous output of a light-pipe under sunny conditions. A light-pipe of length $0.6 \mathrm{~m}$ was supported by a makeshift cardboard frame-surround at both ends. The open end of the light-pipe received direct sun light at an angle of approximately $50^{\circ}$ to the axis of the pipe. The other end of the pipe was covered with the same paper used in the previous example. The highly inhomogeneous nature of the illuminance field leaving the light-pipe is clearly revealed by the pattern of illumination shown on the paper, Figure 5(a).

The following luminance quantities were extracted from the image using Photosphere:

- The mean luminance $L_{s q r}$ of the pixels covering a square region that just encloses the circular light-pipe. This mean luminance results from that due to transmission of light from the pipe, reflectance of light from the camera side of the paper and the luminance contribution from the corner sections of the cardboard surround included in the square region.

- The luminances $L_{c n r}$ of the four corners of the cardboard surround. The mean of the four values $\overline{L_{c n r}}$ is used in the derivation below.

- The luminance $L_{r}$ of a patch of paper placed above the light-pipe on the cardboard surround.

The mean luminance across the paper covering the light-pipe $L_{p}$ can be determined by assuming a circular perimeter for the pipe i.e. the circle is concentric with the square. The mean luminance is determined from:

$$
L_{p}=L_{s q r} \frac{4}{\pi}-\overline{L_{c n r}}\left(\frac{4}{\pi}-1\right)
$$

The mean luminance $L_{s q r}$ of the square region that just bounds the circular pipe was $3840 \mathrm{~cd} \mathrm{~m}^{-2}$. The mean luminance $\overline{L_{c n r}}$ of the four corner sections of cardboard was $711 \mathrm{~cd} \mathrm{~m}^{-2}$. Thus the the mean luminance of the circular section of pipe $L_{p}$ was determined to be $4695 \mathrm{~cd} \mathrm{~m}^{-2}$ using Eqn 7 . This luminance is used in place of $L_{w}$ in Eqn 6, and the area used is now that of the circular paper $\left(0.163 \mathrm{~m}^{2}\right)$. The luminance of the paper due to reflected light $L_{r}$ is subtracted as before (Eqn 6). The luminance coefficient used is now that for direct illumination. There is, of course, no single angle of incidence for light exiting the pipe in the plane of the paper. However, the strongest reflected rays will tend to be those from the sun. These rays will exit the pipe at an angle of incidence similar to that which they entered the pipe with provided that the cylinder is regular. Accordingly, the value at $50^{\circ}$ incidence is used, i.e. $q_{\theta=50}=0.039 \mathrm{sr}^{-1}$ (Figure 3). This gave a luminous output for the light-pipe of 9107 lumens. 
The complex illuminance field at the pipe exit is shown in Figure 5. A logarithmic mapping is used in the false colour image; the scale covers the range four thousand to two million lux. The narrow regions of intense illumination (i.e. "hotspots") are readily apparent. In recent papers by Swift et al and Kocifaj et al, methods to predict the degree and distribution of luminous flux (including hotspots) at the exit aperture of cylindrical light pipes were described $[6,7]$. In the first of these papers, the accuracy of the predicted pattern was assessed by comparing low dynamic range photographs of the illumination field at the exit aperture with those from simulated distributions, i.e. a qualitative comparison was made of the patterns produced. Cylindrical mirrored light pipes $25 \mathrm{~mm}$ in diameter were used in the experiments [6]. The second paper presented a theoretical formulation and gave example output in the form of predicted contours showing the luminous flux emerging from the light pipe [7]. The TIP-HDRI technique described here allows measurements to be carried out on a quantitative basis and for full-size light-pipes, improving greatly on the method used by Swift et al. Kocifaj et al do not present any comparisons of their model output with measurements. The new method presented here would serve equally well as a test of their theoretical formulation.

A significant factor in any deviation between theoretical light-pipe performance and that of an actual installation is likely to be due to the difference between the 'perfect' cylinder geometry used in the theoretical model and the 'imperfect' geometry that occurs in an actual installation. It is unlikely that such effects could be faithfully reproduced using scaled-down version of light-pipes.

\subsection{Measurements of the direct and diffuse horizontal components of global illuminance}

Here the TIP-HDRI technique is used to measure the horizontal components of direct and diffuse illuminance. For this the camera is placed in an open top box with rigid sides (e.g. wood) and the lens directed upwards. The box should should ideally be black inside. However since the rear-side component of reflected luminance is determined and corrected for, the reflectivity of the sides should not matter too much provided that the finish is diffuse. The camera is loosely secured to the base of the box with a "tacky" fixer and the open top is covered with the same calibrated paper used in the previous examples. A lead from the camera is fed through to the controlling laptop computer, Figure 6. The camera lens should have been adjusted in advance to focus on the paper once it is in place. With the paper secured over the opening of the box, a small coin is placed around the centre of the camera's field of view. A stick is positioned to cast a shadow over the central area of the field of view. The automated HDR capture sequence is carried out as before. An illuminance meter was used to record measurements of global horizontal illuminance and diffuse horizontal illuminance by using another stick hand-held to shield direct sun. Note that measurements were recorded in an obstructed area so the contribution of diffuse illumination to the global 
value was less than might be expected for unobstructed settings. This, of course, has no bearing on the outcome of the demonstration.

A small number of measurements were taken to demonstrate proof-ofprinciple (as with all the other examples in this paper, experiments were carried out when time allowed and on an ad hoc basis). The following luminances were derived from the HDR image: the luminance $L_{g}$ produced by the incident direct and diffuse light; and, the luminance $L_{i}$ produced by the diffuse light only. These were simply the uncorrected luminances $L_{g}^{\prime}$ and $L_{i}^{\prime}$ read directly from the HDR image minus the contribution of luminance reflected from inside the box $L_{r}$, i.e that measured "under" the coin, Figure 6 . Next, the luminance $L_{\theta}$ resulting from the contribution of direct sunlight only is simply $L_{g}-L_{i}$.

With the luminances $L_{i}$ and $L_{\theta}$ determined, the associated illuminance values were calculated using, respectively, the luminance coefficients for diffuse and direct illumination. The incidence angle for direct (i.e. sun) illumination was the determined from the sun altitude rounded to the nearest $10^{\circ}$. The HDRI-derived values alongside measured values are shown in Table 1. No summary statistics are given since only a handful of measurements were taken, however from the individual values it seems likely that the typical divergence between measured and HDRI-derived values will be less than $10 \%$. For photometric measurements this must be considered good agreement.

\subsection{Speculative applications of TIP-HDRI}

This new approach lends itself to a wide range of application areas, both in the refining of existing measurement practices and in opening up new possibilities. In addition to those described above the following are noted:

- The deposition of dust and atmospheric pollutants on windows is known to reduce the overall transmittance [8]. Patterns of deposition tend to be inhomogenous thus TIP-HDRI would provide a more reliable means to quantify attenuation than spot measurements. Close inspection of the images in Figure 4 reveals the patterns of rain-streaked dirt reducing the flow of light through the window.

- In addition to large scale shading structures such as deep window reveals and brise soleil, TIP-HDRI could be used to quantify the bulk transmission values of arbitrarily complex fixtures and finishes such as venetian blinds, fabrics, fritted glass, perforated sheets, etc.

- Calibration of bidirectional transmission distribution function (BTDF) models of non-standard glazing materials for simulation. Determining BTDF data requires highly specialised apparatus, e.g. goniophotometer. Great care must be exercised to faithfully resolve the specular components of transmitted light (i.e. peaks in the BTDF) [9]. Measurements of the total transmitted light under controlled conditions could be obtained using TIP-HDRI. Reproducing the measurement scenario in the simulation, and then comparing the values of total transmitted 
light will offer independent part-verification of the BTDF model and/or provide normalisation values.

These are only some of the possible applications that the authors have envisaged.

\section{Discussion}

\subsection{A comparison of TIP-HDRI against standard ap- proaches}

The standard approaches to determining the luminous flux from an optical system require use of either an integrating sphere or a goniophotometer. Either can be considered a 'benchmark' method since they are both the product of many years development and refinement, are capable of high accuracy and have known error characteristics. However, neither method is suitable for measuring the luminous output of windows or light-pipes for reasons given below.

\subsubsection{Integrating sphere alternative}

To get reliable results from an integrating sphere, a certain ratio between the entrance port diameter and the sphere's diameter must be guaranteed, typically of the order of one to five $[10,11]$. This means that the appropriate integrating sphere for a reasonably small window of area $1 \mathrm{~m}^{2}$ would require an integrating sphere of diameter $5 \mathrm{~m}$. The largest integrating spheres that one can find in research institutes are however rarely larger than $3 \mathrm{~m}$ in diameter. In addition, the information provided by an integrating sphere measurement is the overall light transmission coefficient, so no information about the distribution of illuminance over the window area could be obtained.

Alternatively, one could "scan" the window area with a smaller integrating sphere, thus both avoiding the need for impossibly huge spheres and allowing the user to assess how light transmission varies over the window, and hence how illumination varies on the other side (for a non-scattering, non- "light-redirecting" window glazing).

Once the analyzed area gets close to the window area a pixel of the HDR approach would cover, the results would be comparable in spatial resolution while the integrating sphere approach would theoretically be more accurate, since the paper is not a perfect diffuser (see Appendix).

However, the integrating sphere approach would not only be much more cumbersome in terms of equipment and procedure, but it would be immensely more time-consuming. Sky conditions, and more importantly, patterns of direct sun illumination would change significantly over the duration of the scan. The only way to get the measurement time down is to increase the analyzed area, which thus requires to increase the size of the integrating sphere and decrease the quality of information (lower spatial resolution). It is hard to conceive a practical device for quickly scanning large areas 
with an integrating sphere that does not require massive engineering with an associated huge cost.

\subsubsection{Goniophotometer alternative}

To analyze the transmitted light distribution and potentially separate its direct from its diffuse component, one would require a "portable" goniophotometer (so it can be brought inside built spaces) suitable for huge samples (the size of a window).

Existing goniophotometers, whether based on a scanning approach or on a video-based approach combined with a projection principle (these approaches and existing devices are described in [12]) typically only allow samples of dimensions up to about $0.1 \mathrm{~m}$ to $0.4 \mathrm{~m}$ because the investigated sample area has to be considered a point compared to the distance at which light is detected. To measure the flux through a window with a maximum dimension of, say, $2 \mathrm{~m}$ would require a distance of more than $10 \mathrm{~m}$ between the window and the photometer. Thus to analyze a whole window, the size of such goniophotometers would be totally impractical since the volume of space 'swept-out' by the sensor would be much larger than the available indoor space. A theoretically possible exception to this rule would be the goniophotometer proposed by Breitenbach in the late nineties for bidirectional spectroradiometric measurements [13]. The existing prototype of that device, now owned by the the Technical University of Denmark, is unsuited to samples larger than $0.09 \mathrm{~m} \times 0.09 \mathrm{~m}$ and was designed to handle one incident direction at a time. It is not suited to a complex distribution of incident light, and is not at all portable. Potentially, however it could allow a high directional accuracy to be achieved while keeping a large investigated sample area by first focusing the transmitted light with a very large off-axis parabolic mirror onto the end of an optical fiber bundle, whose role is to strongly limit the possible divergence around a given direction.

But while this theoretically feasible option would be incredibly cumbersome to use in an indoor space (maybe impossible depending on the space restrictions), its major draw-back would also be the very long measurement time required to scan each emerging direction with such large mechanical elements. To get an idea, the estimated measurement time for the existing device was 5 to 10 hours per incident direction (this also included a spectral analysis of transmitted light).

The TIP-HDRI approach eliminates the need to scan the emerging light distribution thanks to a somewhat uniform redistribution of light after transmission through the paper, while keeping a known relationship between transmitted luminances and incident illuminance. By diffusing the incoming light through the paper, the information about the transmission directions is lost, though, which would not be the case in the above-described, theoretical goniophotometric approach. 


\subsection{Validation issues and further work}

Rigorous demonstrations of the absolute accuracy of TIP-HDRI will be rather more demanding than any of the experiments described in the application examples section. Comparison between TIP-HDRI derived values and independent measurements is given in Section 4.3. We hesitate at this stage to call an experiment with such a small number of measurements a validation. Nonetheless, the small deviation between the TIP-HDRI derived components of illuminance and those measured with a calibrated meter is certainly encouraging (Table 1), and does provide a test of the fundamental basis of the technique. Extrapolating to the case of measuring flux through a window, we surmise that the principle obstacles to achieving similar accuracy are the currently un-quantified effect of backscatter between the paper and the glazing and the uncertainty resulting from using a single luminance coefficient (rather than separate diffuse and direct values). A third factor to consider in a rigourous validation is the spectral composition of the transmitted light.

\subsubsection{Backscatter}

Backscatter occurs when light reflected from the paper is re-directed back onto the paper by reflection at the glass-air interfaces. This could result in a systematic over-estimation of luminous flux of the order of $10 \%$ or perhaps more for clear double glazing. Our first thought was to quantify this effect by comparing TIP-HDRI derived illuminances with measurements for cases with and without glass. The limited testing without glass (i.e. determination of direct and diffuse components of horizontal illuminance) showed good agreement with measurements. However, the case with glass would not be so straightforward to carry out since the sensor 'head' of an illuminance meter is itself reflective and therefore also prone to backscatter effects when taking measurements against glass. An arrangement that might work for quantifying the backscatter effect resulting from direct beam radiation is to have the paper and glass held in a non-reflective tube. Since the paper is essentially a diffuse reflector of light, increasing the separation between the glass and the paper will progressively decrease the amount of the backscattered light. Thus the magnitude of the backscatter could be determined for normal incidence direct beam. The direct beam radiation would need to be collimated to ensure constant illumination over the scale of movement of paper or glass in the tube. A more elaborate experimental arrangement would be needed for incidence angles other than normal, and the approach will not be effective for diffuse illumination. Reducing the overall magnitude of the backscatter effect could be achieved by using paper with a printed, non-reflective pattern. The regions of the paper that do not have the pattern would need to be of sufficient size that the corresponding pixels in the HDR image could be reliably identified. Additionally, there would need to be sufficient coverage of the un-inked paper so that interpolation could reliably estimate the flux absorbed by the non-reflective pattern. This approach would probably work well for reducing the backscatter from highly reflective housings where the reflecting surfaces are at some distance away from the paper, as would be 
the case if TIP-HDRI were used to measure the flux from a luminaire in a box-like housing. However, it would be of limited use where the paper needs to be against the reflecting surface (i.e. the glass) since then significant components of the backscatter have a highly localised effect, i.e. the 'first order' reflection at the air-glass interface immediately adjacent to the paper. Thus the contribution of the 'first order' backscatter reflection is much the same as it was with the un-inked paper. Note that 'open' light-pipes (as described in Section 4.2) are not subject to backscatter effects.

\subsubsection{Separating direct and diffuse components of illumination}

To obtain the greatest accuracy possible with TIP-HDRI it may be necessary to separate total illumination into direct and diffuse components and apply the respective luminance coefficient values for the derivation of illuminance from luminance. This was simple to do for the example application given in Section 4.3 since it was reasonable to assume uniform diffuse illumination. However, for the window examples the separation turns out to be rather more involved since the diffuse component of illumination can vary significantly across the window. When this occurs it is not a straightforward matter to separate the components using standard image-processing techniques such as segmentation by thresholding. The variation in the recorded luminance of the paper across small spatial scales caused by the inhomogeneity of the paper further confounds the application of off-the-shelf segmentation techniques. More involved methods based on sampling the gradients in the image have shown promise, but further work in this area is required. Selecting the most appropriate luminance coefficient for the determination of flux through the light pipe is not a straightforward matter and may be complicated further if there is no single angle of incidence for direct illumination. The integrating sphere method could be used to validate TIP-HDRI measurements of the flux from light pipes. Controlled illumination conditions would be needed and, for the reasons given in Section 5.1, the diameter of pipe that could be tested would be constrained by the size of the integrating sphere. A validation method using an independent HDR method which is not subject to the directional sensitivity of the luminance coefficient has been proposed in a companion paper to follow. These uncertainties notwithstanding, the method described in Section 4.2 nevertheless delivers measurements on fulldiameter light-pipes under real world conditions that are largely unobtainable using the standard methods.

\subsubsection{Spectral considerations}

Whilst the spectral reflectivity of paper is reasonably constant over the visible range, the same may not be true for the transmission of light since scattering is known to be wavelength dependent. Furthermore, there may be a complex interaction between the spectral distribution of the light source, the spectral selectivity of the paper (if significant) and the spectral response of the camera CCD and the illuminance sensor used for validation. The significance or 
otherwise of spectral effects for the technique described here has yet to be quantified.

\subsubsection{Summary}

It is evident from the above that determining the absolute accuracy of TIPHDRI derived illuminances to a high degree of precision presents a challenging program of work. The authors have envisaged experiments that should provide rigourous tests of TIP-HDRI for some of the application areas. Tests for full-size light-pipes however are likely to be constrained by the diameter of the available integrating sphere. The authors intend to carry out a validation study in the near future.

\section{Conclusion}

The TIP-HDRI method presents numerous possibilities to measure luminous flux in a wide variety of application areas. Measurement scenarios that are either impractical or unattainable using the standard methods are rendered achievable. The technique requires only modest investment in equipment. Furthermore, compared to standard laboratory methods, the demands of time and expertise are not great. The technique is very new and has not yet been fully explored nor subjected to rigorous testing. The small number of preliminary test results are however very encouraging and the technique has the potential to revolutionise the practice of light metrology for certain applications. The authors will continue to expand and refine the TIP-HDRI technique, and also to seek more definitive means of validation.

\section{Acknowledgements}

The HDR capture program HDRcapOSX and the HDR image browser Photosphere were both created by Greg Ward of Anyhere Software. They are both MAC OS X applications and are freely-available from Anyhere Software [5]. The authors thank Joe Lynes and Steve Selkowitz for suggestions regarding backscatter and Nicholas Gayeski for his help in performing the set of measurements carried out at MIT. We thank Monodraught Limited for supplying the light-pipe. We gratefully acknowledge suggestions from the referees and the Technical Editor to help 'streamline' the paper.

\section{References}

[1] M. N. Inanici. Evaluation of high dynamic range photography as a luminance data acquisition system. Lighting Research and Technology, 38(2):123-134, 2006.

[2] M. Fontoynont and V. Berrutto. Daylighting performance of buildings: Monitoring procedure. Right Light 4, 2:119-127, 1997. 
[3] Commission Internationale de l'Eclairage. Radiometric and photometric characteristics of materials and their measurement. CIE 38 (TC-2.3), 1977 .

[4] M. Andersen. Innovative bidirectional video-goniophotometer for advanced fenestration systems. PhD thesis, EPFL, 2004.

[5] G. Ward. Anyhere Software http://www.anyhere.com/.

[6] P. D. Swift, G. B. Smith, and J. Franklin. Hotspots in cylindrical mirror light pipes: description and removal. Lighting Research and Technology, 38(1):19-28, 2006.

[7] M. Kocifaj, S. Darula, and R. Kittler. Holigilm: Hollow light guide interior illumination method - an analytic calculation approach for cylindrical light-tubes. Solar Energy, 82(3):247-259, 2008.

[8] S. Sharples, L. Stewart, and P. Tregenza. Glazing daylight transmittances: a field survey of windows in urban areas. Building and Environment, 36(4):503-509, 2000.

[9] M. Andersen and J. L. Scartezzini. Inclusion of the specular component in the assessment of bidirectional distribution functions based on digital imaging. Solar Energy, 79(2):159-167, 2005.

[10] Commission Internationale de l'Eclairage. Practical methods for the measurement of reflectance and transmittance. CIE 130, 1998.

[11] Commission Internationale de l'Eclairage. The measurement of luminous flux. CIE 84, 1989.

[12] M. Andersen and J. de Boer. Goniophotometry and assessment of bidirectional photometric properties of complex fenestration systems. Energy and Buildings, 38(7):836-848, 2006.

[13] J. Breitenbach and J. L. J. Rosenfeld. Design of a photogoniometer to measure angular dependent optical properties. Proceedings of International Conference on Renewable Energy Technologies in Cold Climates, Ottawa, Canada, pages 386-391, 1998.

[14] IESNA. Lighting Handbook Ninth Edition, New York, 2000.

[15] M. Andersen. Validation of the performance of a new bidirectional video-goniophotometer. Lighting Research and Technology, 38(4):295$311,2006$. 


\section{Appendix - The 'expert' measurements}

This Appendix describes in detail the measurements and data analysis carried out by Andersen at MIT, i.e. the 'expert' set. The measurements were conducted in a $4 \mathrm{~m} \times 4.5 \mathrm{~m}$ black chamber and the optical equipment used to perform them included:

- a Minolta LS-110 luminance-meter

- a LMT illuminance-meter Pocket-Lux 2B

- two Labsphere integrating spheres (Spectraflect coating): the 3P-GPS040-SF (4 in. diameter (about $100 \mathrm{~mm}$ )) and the RTC-060-SF (6 in. diameter (about $150 \mathrm{~mm})$ )

- a tungsten-halogen light source (KI-120 Kohler Illuminator, 120 W)

- various holders and positioning accessories (optical rail, posts, carriers, etc.).

A schematic of the experimental set-up is illustrated in Figure 7a: the light beam produced by the tungsten-halogen source was focused on the 1 in. (25 mm) entrance port of the integrating sphere and the ink-jet paper (or the illuminance-meter sensor) placed flush with a 1.25 in. (about $32 \mathrm{~mm}$ ) exit port located 16 degrees off axis from the incoming beam. The incoming light was therefore first "integrated" inside the sphere so as to illuminate the paper or the illuminance-meter sensor in a diffuse and uniform way. Note, to not clutter the schematic, the entry port is shown at $90^{\circ}$ to the incoming beam (Figure 7a).

As expected, there was high uniformity of the spot at the exit port: relative variations of less than $1 \%$ and $3 \%$ were observed in terms of illuminance and luminance respectively. As far as relative fluctuation of a given reading was concerned, it was observed to be almost negligible: about $0.1 \%$ for luxmeter readings and less than $1 \%$ for luminance-meter readings.

To keep a consistent approach with direct illumination measurements, five illuminance readings were taken (for slightly shifted luxmeter positions over the exit port area) both before and after luminance measurements were performed, and for each luminance-meter position $\left(0^{\circ}, 10^{\circ}, 30^{\circ}, 50^{\circ}, 60^{\circ}\right.$ and $\left.70^{\circ}\right)$, five readings were taken for slightly shifted areas over the spot on the paper.

Stray light (and any "back reflection" off of the paper) was again checked for luminance-meter positions $0^{\circ}$ and $70^{\circ}$, and proved to be negligible.

\section{Direct and diffuse illumination}

Because of the non-uniformity of the spot mentioned earlier for direct illumination, ratios between luminance $L\left(\mathrm{~cd} \cdot \mathrm{m}^{-2}\right)$ and illuminance $E$ (lux) values at each "spot location" were considered individually. The obtained ratios $q=L / E\left(\mathrm{sr}^{-1}\right)$ were then averaged to get a unique $q$ value for each illumination and viewing angle configuration $\left(q_{\mathrm{dir}}\right.$ for direct illumination, $q_{\mathrm{diff}}$ for 
diffuse illumination), whose associated experimental errors include both the variation of these ratios (from $2.3 \%$ to $9.6 \%$ depending on the configuration) and the fluctuations in luxmeter and in luminance-meter readings.

The experimental results for diffuse and directional $q$ were shown in Figure 3 together with those from the 'novice' set. In Figure 8, the data obtained at MIT for varying luminance-meter positions are given for both illumination types (direct and diffuse). From these graphs, several statements can be made about the diffusing properties of the ink-jet paper. If the ink-jet paper was a perfect diffuser (following the theoretical Lambertian model), one would expect constant $q_{\text {dir }}$ values for varying incident angles and, for each incidence, constant $q_{\text {dir }}$ values for varying luminance-meter angles. Although this is not strictly observed on the curves of Figures 3 and 8, it can be noticed that:

- Whatever the illumination type (direct, diffuse) and incident direction $\left(0^{\circ}, 30^{\circ}, 60^{\circ}\right)$, the luminance distribution remains the same in relative terms: we observe a gradual decrease of the ratio $q=L / E$ from the normal to the paper towards the more grazing luminance-meter angles, without any luminance increase around the direct transmission direction for non-normal direct illumination.

- This gradual decrease remains identical for all incident angles even though the overall transmission decreases with increasing incident angles.

- Up to 50 degrees off normal both for incoming light and for transmitted light, $q$ values remain within $20 \%$ of their value along the normal to the paper, independently of the illumination type.

The markers labelled " $2 \mathrm{x}$ ", " $3 \mathrm{x}$ " and " $5 \mathrm{x}$ " in Figure 8 indicate the angular size of an object (e.g. window) when the camera is placed at a distance 2, 3 and 5 times the maximum dimension of the object. The last in the list is of course the "five times rule" recommended for the photometry of linear light fixtures [14].

These observations allow us to conclude that the ink-jet paper shows sufficiently diffusing transmission properties for the position of the camera for HDR analysis to be unconstrained as long as it remains within $50^{\circ}$ off the normal to the window, and for the paper to be appropriate for a reliable investigation of the impinging light, whatever its spatial distribution.

First, a series of transmission measurements was realized for direct illumination, for which the collimated beam produced by the tungsten-halogen source was used.

The paper holder was placed at an appropriate distance from the source to obtain a uniform spot and the luminance-meter positioned on the other side of the paper, at about a meter from it so as to ensure a proper focusing. A schematic of the arrangement is shown in Figure $7 \mathrm{~b}$. The paper was rotated by increments of 10 degrees, and luminance-meter readings taken along the normal to the paper, as well as, for some incident directions, along directions off normal, whose angle to the normal varied between $10^{\circ}$ and $70^{\circ}$ : 
- For incident direction $0^{\circ}$ (normal incidence), luminance-meter measurements were performed at $0^{\circ}$ (normal viewing angle), $10^{\circ}, 20^{\circ}, 30^{\circ}, 40^{\circ}$, $50^{\circ}, 60^{\circ}$ and $70^{\circ}$.

- Because of the very gradual variation in luminance values observed along the above set of emerging directions, larger angular increments were chosen for incidences $30^{\circ}$ and $60^{\circ}$, where luminance measurements were taken at $0^{\circ}, \pm 10^{\circ}, \pm 30^{\circ}, 50^{\circ}, \pm 60^{\circ}$ (only $+60^{\circ}$ for incidence $30^{\circ}$ ) and $70^{\circ}$.

- For the other incident directions $\left(10^{\circ}, 20^{\circ}, 40^{\circ}, 50^{\circ}, 70^{\circ}\right.$ and $\left.80^{\circ}\right)$, luminance-meter measurements were only performed along the normal to the paper $\left(0^{\circ}\right)$.

The "minus" angular values (corresponding to $10^{\circ}, 30^{\circ}$ and $60^{\circ}$ off normal in the other direction) mentioned in the above list were added so as to be able to appreciate how the luminance varied when the viewing direction approached the direct transmission direction, and thus assess the diffusing qualities of the ink-jet paper in a more extensive way.

Both before and after a set of luminance measurements was completed for a given incident direction, illuminance readings were taken, the paper holder being then replaced by a luxmeter holder, positioned so as to ensure that the sensitive area of the luxmeter was in the same plane as the paper was.

The spot produced by the tungsten-halogen light source being slightly non-uniform (5\% relative variations in illuminance were observed over the spot area, whose diameter is of about $25 \mathrm{~mm}(1 \mathrm{in}$.)), a set of five readings was taken for each configuration, both when taking illuminance measurements and luminance measurements. Each illuminance-meter and luminance-meter reading was associated to a particular area of the spot: the sensing area of the illuminance-meter and the area observed with the luminance-meter were gradually moved from the bottom of the spot to the top of the spot (the variations were much more significant along that vertical line than from left to right).

For each of the five readings, the fluctuation in illuminance or luminance was recorded and found out to be very small: in average, $0.05 \%$ for illuminance-meter readings and $1.4 \%$ for luminance-meter readings for all configurations.

In order to check whether stray light had a significant impact on the obtained luminance values, "back reflection" measurements were performed in the following way: an perfectly opaque layer was placed in front of the ink-jet paper with the light source switched on (double layer of velvetine with a paper surface facing the incident beam so as to account for light reflected off of the ink-jet paper) and luminance-meter readings were taken at $0^{\circ}$ and $70^{\circ}$ for incident directions $0^{\circ}, 30^{\circ}$ and $60^{\circ}$.

The obtained luminance values varied between 0 and $0.02 \mathrm{~cd} \cdot \mathrm{m}^{-2}$ i.e. were much smaller than the other readings' own fluctuations. It was hence concluded that stray light was negligible and that the transmission measurements were not affected by any significant back reflection off of the paper. 


\section{Verification of the diffusing properties of paper}

From the set of $q$ values obtained for a given incidence direction, one can estimate the value of the directional-hemispherical transmittance $\tau_{\mathrm{dh}}$ based on an integration of the BTDF (i.e. $1 / q)$ values $\left(\mathrm{sr}^{-1}\right)$ corresponding to that incident direction, assuming that the paper is isotropic (no dependence on azimuth angles) [3]. The integration method is described in [15]. This calculated $\tau_{\text {dh }}$ value can then be compared with the one obtained through an integrating sphere measurement for the same incident direction. This experiment was realized for normal incidence by calculating the ratio between the light flux detected inside the sphere with the paper placed flush with the entrance port and the light flux detected without any paper, keeping the same incident direction.

The resulting values for paper transmission $\tau_{\mathrm{dh}}$ under normal incidence were close: $\tau_{\text {dh-meas }}=14.6 \% \pm 0.03 \%$ for measured paper transmission; $\tau_{\text {dh-calc }}=13.4 \% \pm 0.04 \%$.

The integrating sphere measurement was not repeated for other incident angles but the $\tau_{\text {dh-calc }}$ values for incidences $30^{\circ}$ and $60^{\circ}$ were found to be $12.4 \% \pm 0.04 \%$ and $10.4 \% \pm 0.03 \%$ respectively.

Likewise, one can estimate the value of the hemispherical-hemispherical transmittance $\tau_{\text {hh }}$ based on an integration of the BTDF values $\left(\mathrm{sr}^{-1}\right)$ obtained under diffuse illumination. In this case, the resulting $\tau_{\text {hh-calc }}$ value was $14.1 \% \pm 0.04 \%$.

Altogether, for the HDR analyzes, one can consider that the transmission coefficient of the paper, whether for normal direct or diffuse illumination, is of about $14 \% \pm 0.5 \%$, and that it decreases by about a percent when the incident angle increases by $15^{\circ}$ up to $70^{\circ}$. 


\begin{tabular}{rrrrrr}
\hline \multicolumn{2}{c}{ Obstructed diffuse [lux] } & \multicolumn{3}{c}{ Direct sunlight [lux] } \\
Measured & TIP-HDRI & (error) & Measured & TIP-HDRI & (error) \\
\hline 9400 & 8976 & $(-4.5 \%)$ & 66100 & 69448 & $(5.1 \%)$ \\
\hline 10100 & 10271 & $(1.7 \%)$ & 83200 & 82306 & $(-1.1 \%)$ \\
\hline 8360 & 9178 & $(9.8 \%)$ & 66040 & 63827 & $(-3.5 \%)$ \\
\hline 8200 & 8016 & $(-2.2 \%)$ & 56800 & 58064 & $(2.2 \%)$ \\
\hline
\end{tabular}

Table 1: Measured and HDRI derived illuminance values 
(a) Aperture

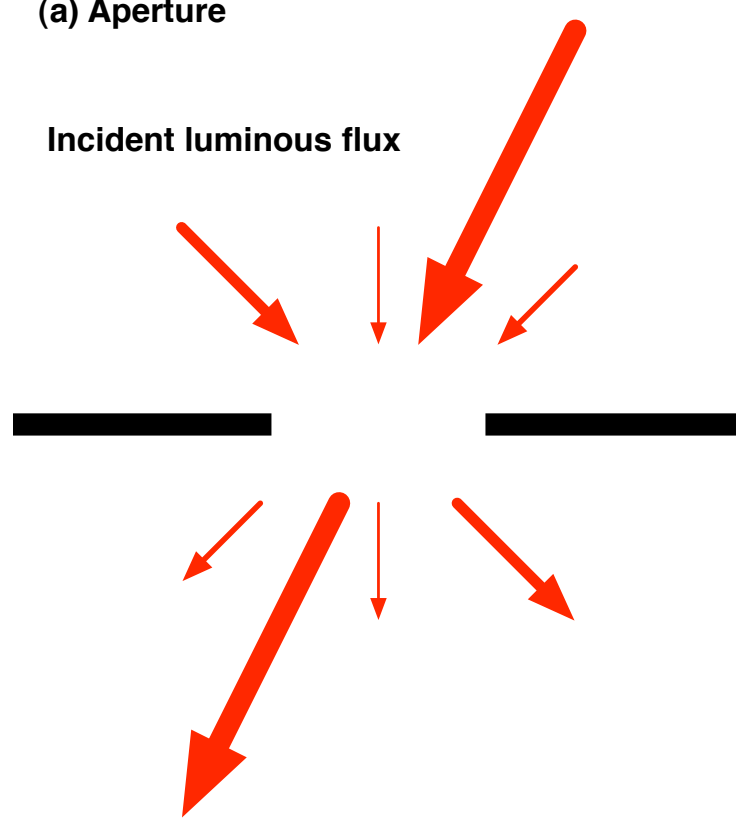

(b) With diffusing material

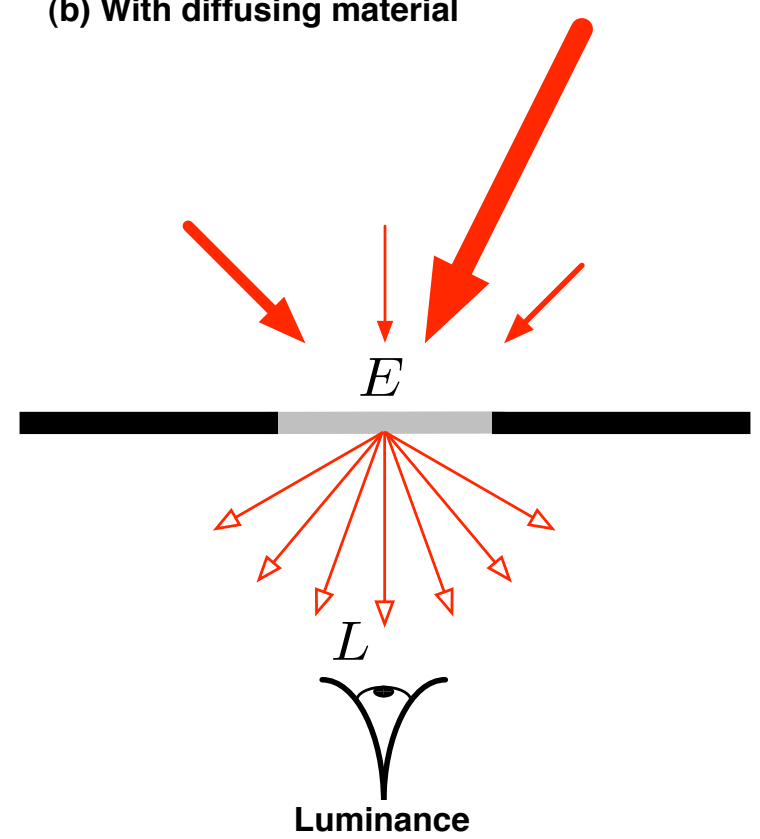

Figure 1: Luminous flux with arbitrary direct and diffuse components passing through a building aperture, e.g. window (a). The incident flux will produce a luminance that can be observed or measured when a suitable diffusing material is placed over the aperture (b) 
(a) Isotropic incident flux

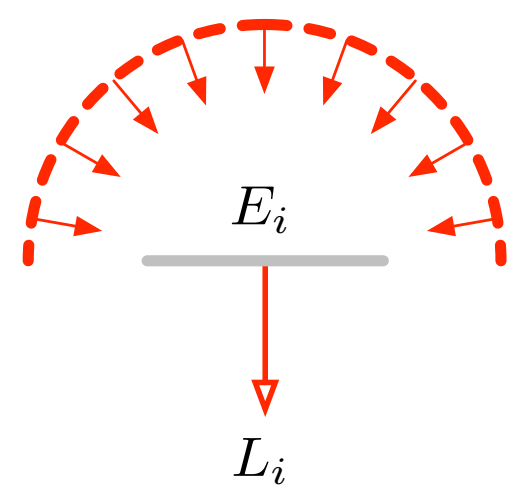

(b) Directional incident flux

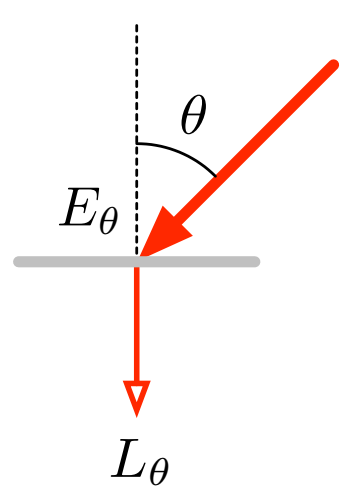

(c) Near-side reflected flux

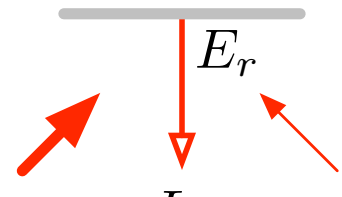

$L_{r}$

Figure 2: Luminance of the diffusing material can result from three sources: (a) incident isotropic illumination, (b) incident directional illumination, and (c) rear-side incident illumination 


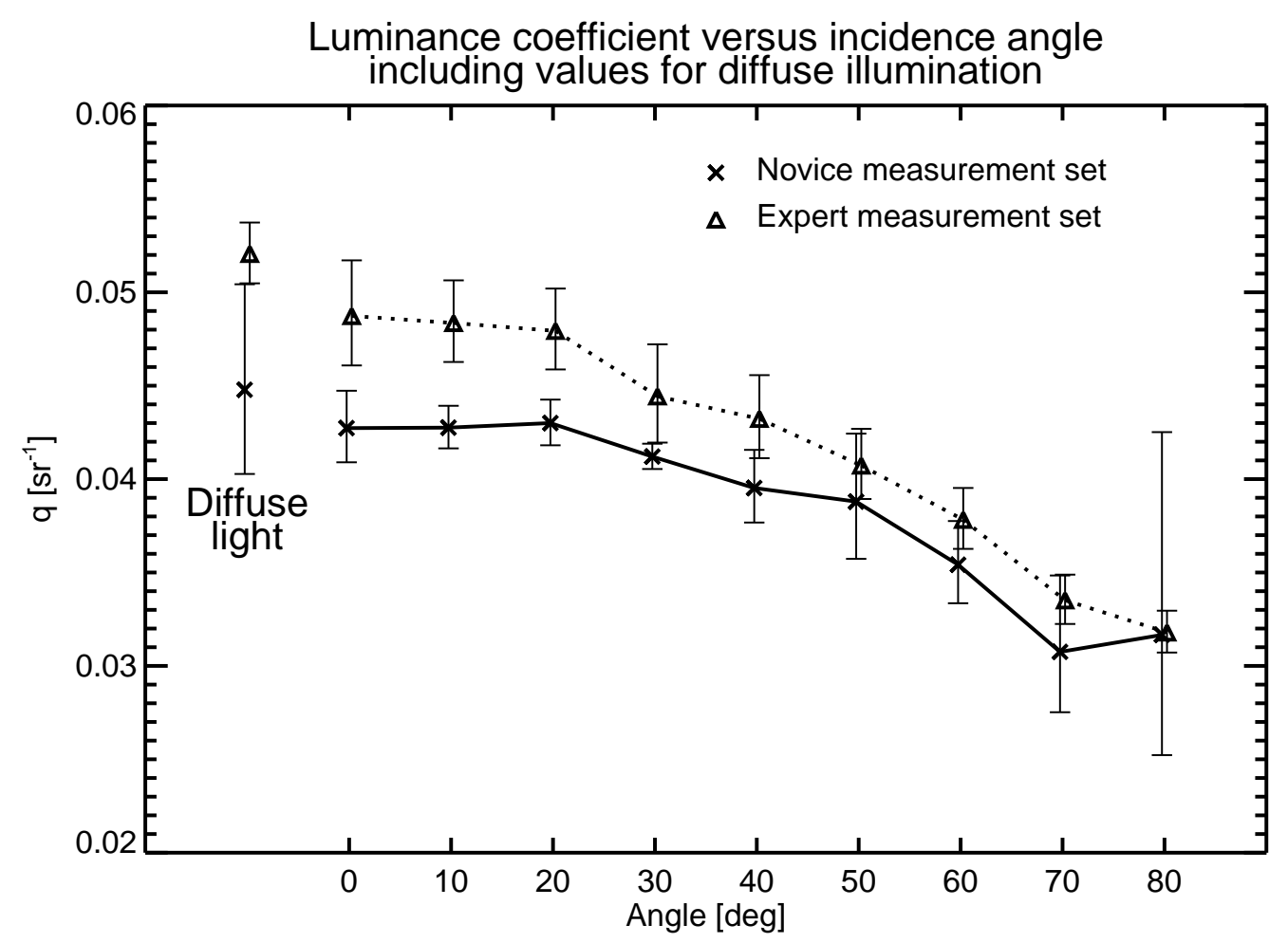

Figure 3: Comparison of novice and expert measurements sets for the luminance coefficient 

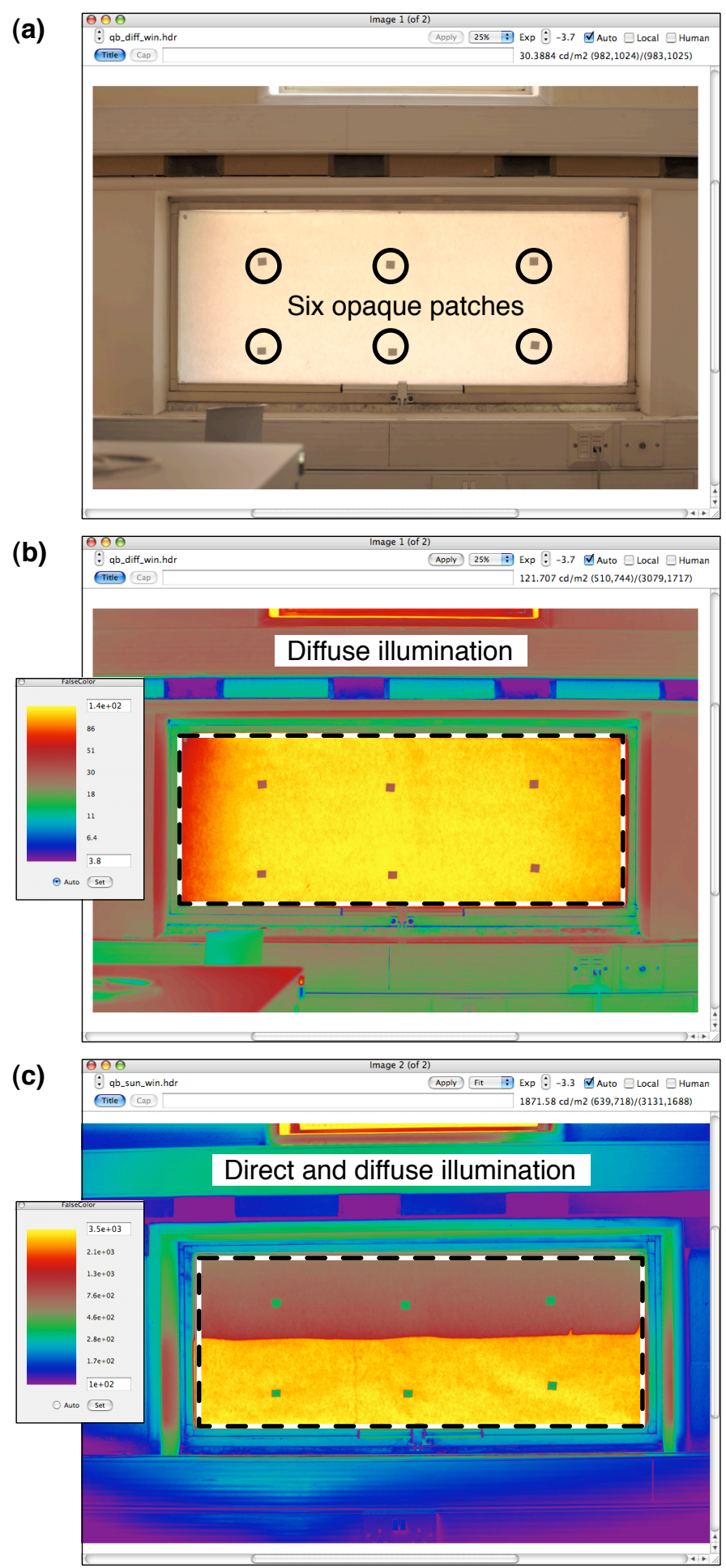

Figure 4: Measurement of the lumen output of a window under diffuse natural illumination and with direct sun. Standard digital camera image with opaque patches identified (a), false-colour HDR images of window under diffuse illumination (b) and with partially-shaded direct sun (c) 


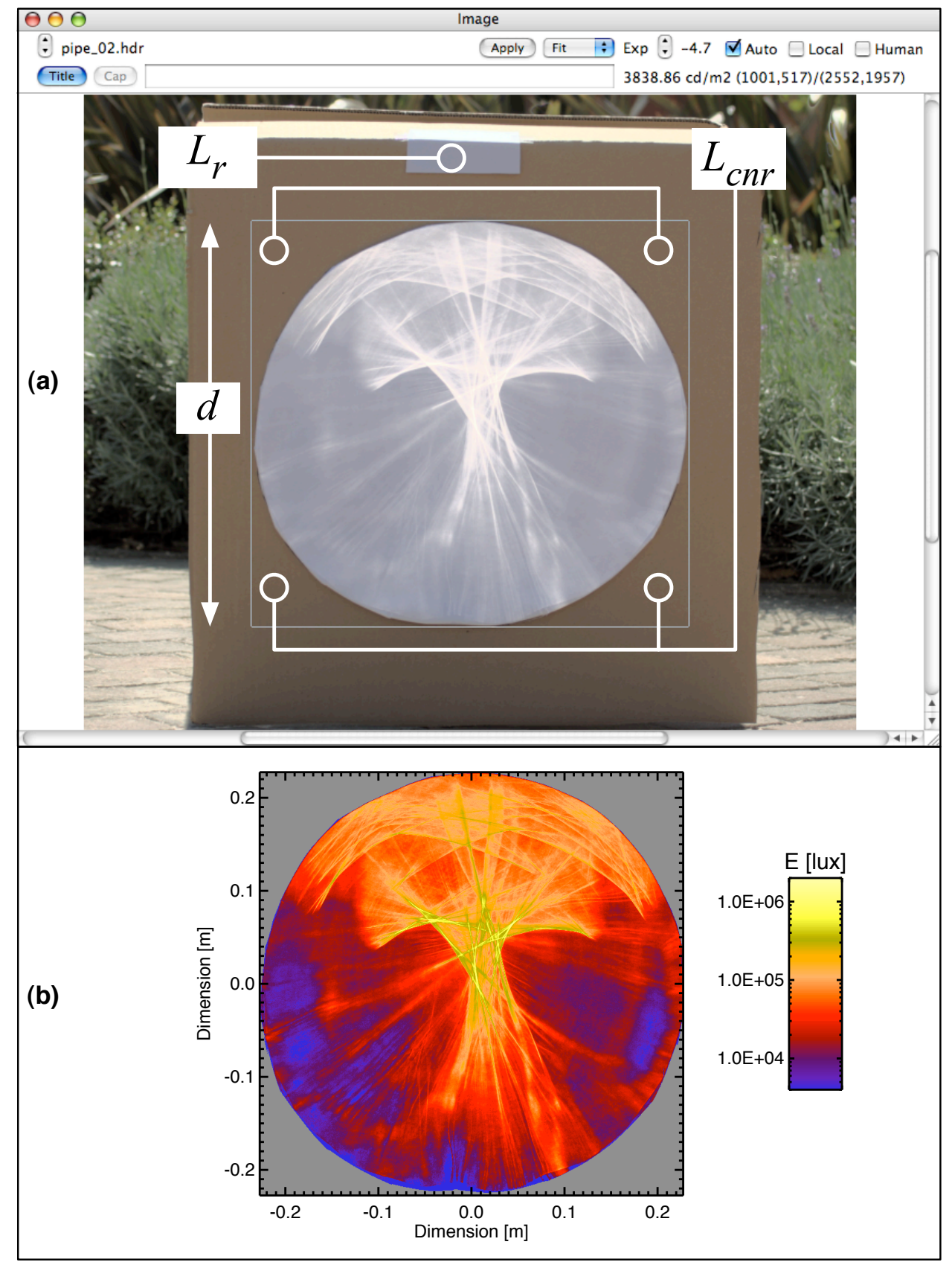

Figure 5: HDR image of the light-pipe with paper placed over the exit aperture displayed in Photosphere (a). The luminance of the paper recorded by the HDR capture was processed to derive the illuminance field at the exit aperture, shown in false-colour using a logarithmic mapping (b) 
(a)
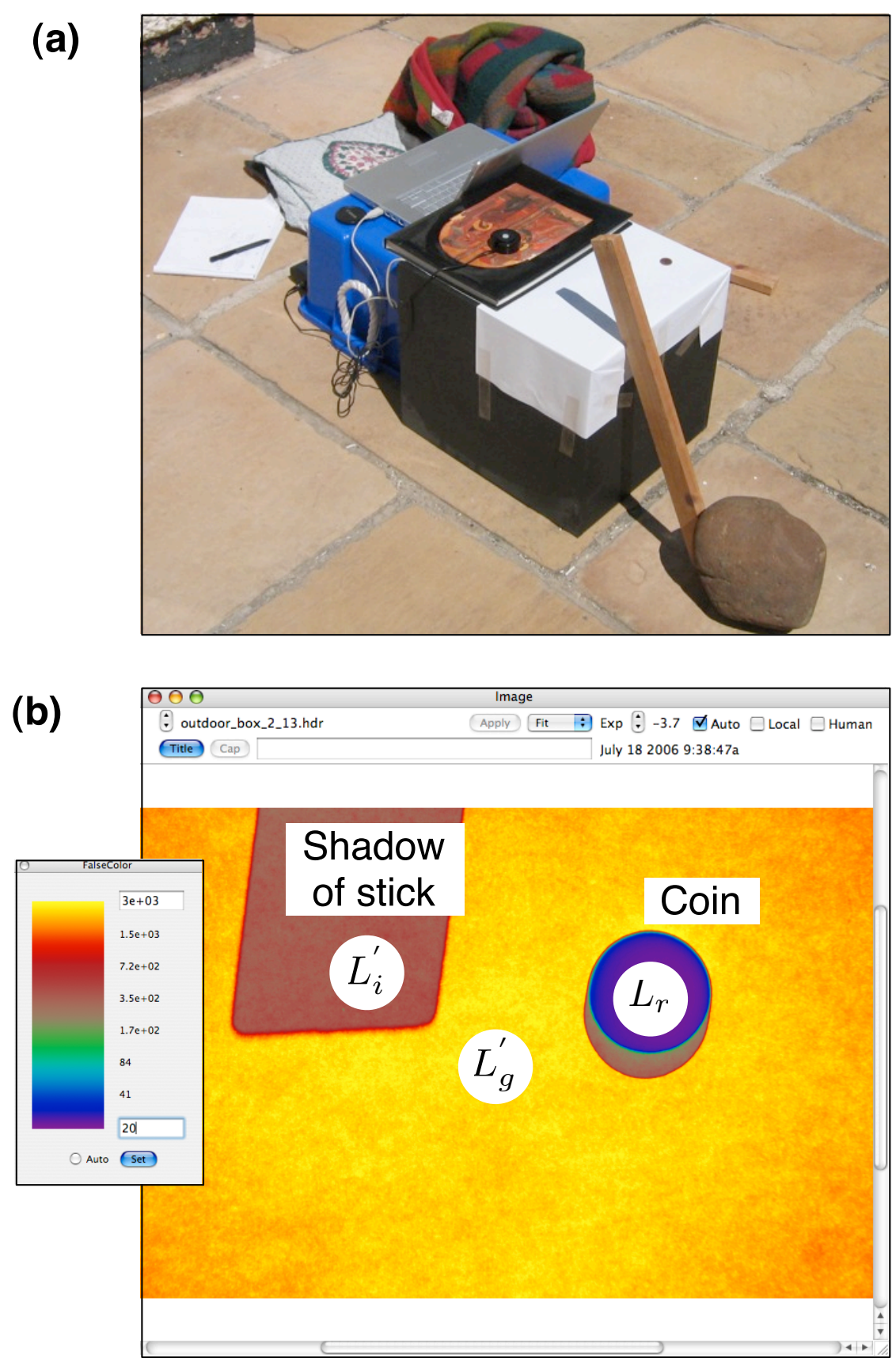

Figure 6: Experimental setup for measuring direct and diffuse components of horizontal illumination (a). The digital SLR is placed inside the box, on the base, facing "up" with the lens focussed on the paper. A laptop computer controls the HDR capture. The resulting HDR image of the paper luminance is shown using false colour in (b) 


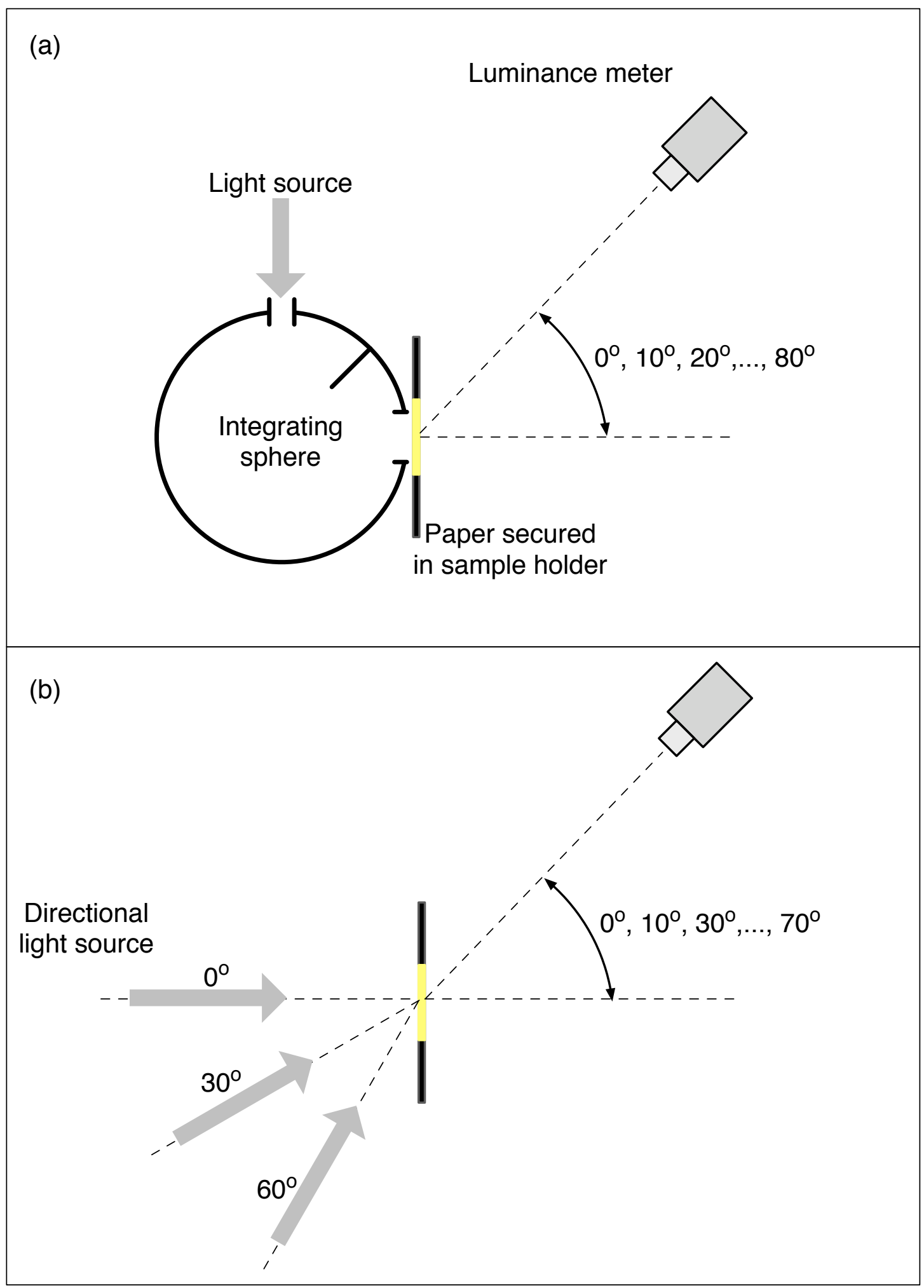

Figure 7: Schematics for the experimental set-up for the 'expert' measurements: (a) diffuse light with integrating sphere; and, (b) directional light 


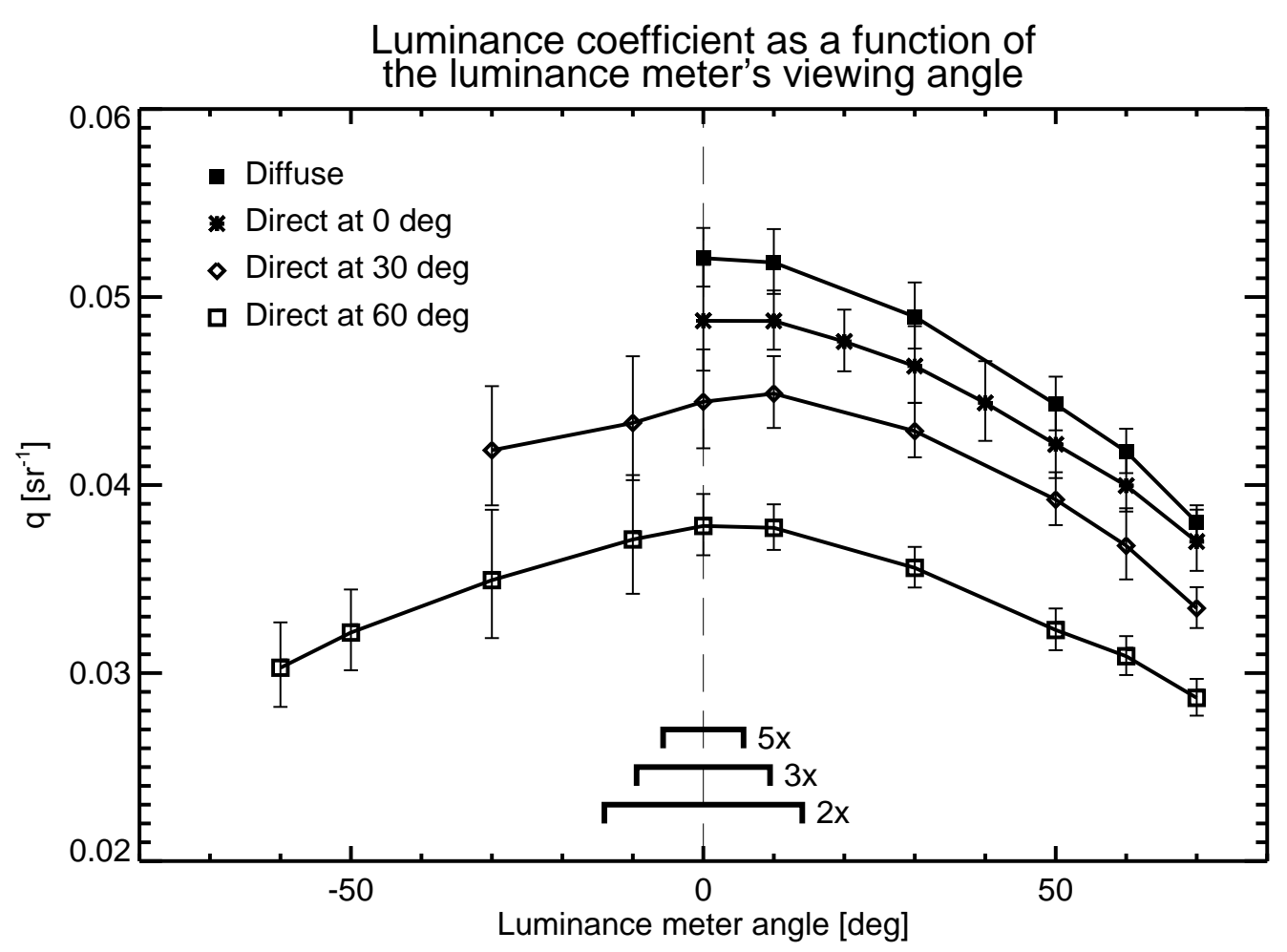

Figure 8: Verification of the paper's diffusing properties: $q$ values as a function of the luminance meter's viewing angle 